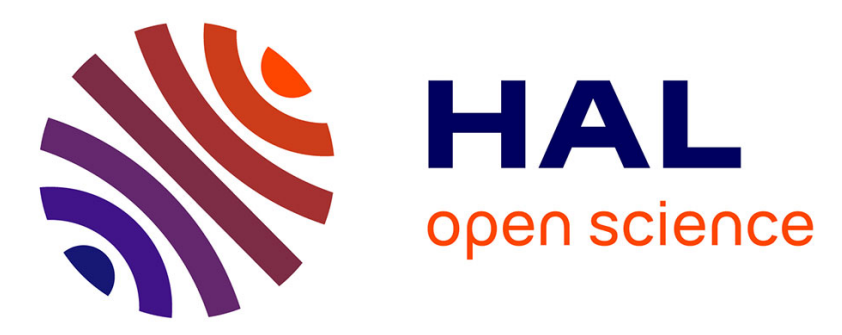

\title{
A combined model predictive control and time series forecasting framework for production-inventory systems
}

Philip Doganis, Eleni Aggelogiannaki, Haralambos Sarimveis

\section{To cite this version:}

Philip Doganis, Eleni Aggelogiannaki, Haralambos Sarimveis. A combined model predictive control and time series forecasting framework for production-inventory systems. International Journal of Production Research, 2008, 46 (24), pp.6841-6853. 10.1080/00207540701523058 . hal-00512994

\section{HAL Id: hal-00512994 \\ https://hal.science/hal-00512994}

Submitted on 1 Sep 2010

HAL is a multi-disciplinary open access archive for the deposit and dissemination of scientific research documents, whether they are published or not. The documents may come from teaching and research institutions in France or abroad, or from public or private research centers.
L'archive ouverte pluridisciplinaire HAL, est destinée au dépôt et à la diffusion de documents scientifiques de niveau recherche, publiés ou non, émanant des établissements d'enseignement et de recherche français ou étrangers, des laboratoires publics ou privés. 


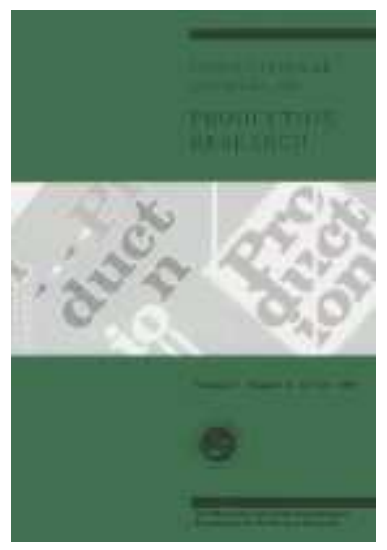

\section{A combined model predictive control and time series forecasting framework for production-inventory systems}

\begin{tabular}{|r|l|}
\hline Journal: & International Journal of Production Research \\
\hline Manuscript ID: & TPRS-2007-IJPR-0134.R1 \\
\hline Manuscript Type: & Original Manuscript \\
\hline Author: & O7-Jun-2007 \\
\hline & $\begin{array}{l}\text { Complete List of Authors: } \\
\text { Aggelogiannaki, Eleni; National Technical University of Athens, } \\
\text { School of Chemical Engineering } \\
\text { Sarimveis, Haralambos; National Technical University of Athens, } \\
\text { School of Chemical Engineering }\end{array}$ \\
\hline Keywords: & $\begin{array}{l}\text { PROCESS CONTROL, INVENTORY CONTROL, PRODUCTION } \\
\text { PLANNING, FORECASTING, NEURAL NETWORKS, GENETIC } \\
\text { ALGORITHMS }\end{array}$ \\
\hline Keywords (user): & MODEL PREDICTIVE CONTROL \\
\hline
\end{tabular}

\section{s scholaroNE" \\ Manuscript Central}




\title{
A combined model predictive control and time series
}

\section{forecasting framework for production-inventory systems}

\author{
PHILIP DOGANIS, ELENI AGGELOGIANNAKI, AND HARALAMBOS
}

SARIMVEIS*

School of Chemical Engineering, National Technical University of Athens, Greece

\begin{abstract}
Model Predictive Control (MPC) has been previously applied to supply chain problems with promising results; however most systems that have been proposed so far possess no information on future demand. The incorporation of a forecasting methodology in an MPC framework can promote the efficiency of control actions by providing insight in the future. In this paper this possibility is explored, by proposing a complete management framework for production-inventory systems that is based on MPC and on a neural network time series forecasting model. The proposed framework is tested on industrial data in order to assess the efficiency of the method and the impact of forecast accuracy on the overall control performance. To this end, the proposed method is compared with several alternative forecasting approaches that are implemented on the same industrial data set. The results show that the proposed scheme can improve significantly the performance of the production-inventory system, due to the fact that more accurate predictions are provided to the formulation of the MPC optimization problem that is solved in real time.

*Corresponding author. Tel.: +30 210 7723237; fax: +30-210-7723138.

E-mail address.hsarimv @ central ntua.or (Hortharimveis).ril: ijpr@lboro.ac.uk
\end{abstract}


Keywords: Process Control, Forecasting, Model Predictive Control, Production planning, Inventory Control, Neural Networks, Genetic Algorithms

1

2

4

5

7

8

9

10

11

12

13

14

15

16

17

18

19

20

21

22

23

24

25

26

27

28

29

30

31

32

33

34

35

36

37

38

39

40

41

42

43

44

45

46

47

48

49

50

51

52

53

54

55

56

57

58

59

60 


\section{Introduction}

During the last decades, industrial goods production has shifted from the local or national level to facilities with global outreach that serve many national markets. This development has put substantial stress on the supply chains of companies, which seek for methodologies that can lead to a better management of performed actions and improve customer service, while reducing cost. This paper will demonstrate the coupling of Model Predictive Control (MPC) with a neural network based forecasting system and investigate its effectiveness on the production-inventory control problem.

MPC was first applied to inventory management by Kapsiotis and Tzafestas (1992), who studied a single manufacturing site problem. Perea-Lopez et al (2003) employed MPC to the management of a multi-level supply chain with multiple products where demand was assumed deterministic. Braun et al (2003) presented a linear MPC methodology for large scale supply chain problems and showed that MPC can handle uncertainties due to model mismatch and forecast errors. Lin et al (2005) presented a Minimum Variance Control system with set points not only for the actual inventory level, but also for the Work-InProcess (WIP) level, while customer demand was expressed by an AutoRegressive Integrated Moving Average (ARIMA) model. Their formulation maintained inventory levels at a desired level, while avoiding the "bullwhip" effect. 
Forecasting plays a central role in the efficient operation of a supply chain, as it provides valuable information on the expected future direction of various factors. Among them, customer demand is perhaps the most important factor to be predicted through a forecasting system. The simplest forecasting method which is very common in typical industrial MPC configurations is to set all demand forecasts equal to the current demand value, assuming that this is available exactly (Mestan et al, 2006). More advanced forecasting methodologies typically produce time series models, which can be classified as linear or nonlinear. Linear models are the most popular ones, due to their simplicity and ease of use. Examples of widely used linear methodologies are the ARIMA models (Box et al. 1994) and the AutoRegressive Moving Average (ARMA) models, which are special cases of the former. The forecast in these two methodologies is produced by linearly combining past values of the time series and past forecast errors. Another popular approach is the Holt-Winters method, which is an exponential smoothing methodology. As such, it uses weighted values of past time series occurrences, where the coefficients decay exponentially with each period, thus giving more weight to recent values and less to more distant ones. Its structure can capture trends and seasonality in data, making it suitable for various types of time series data.

In most methods, including the two mentioned above, the critical parameters of the equations that describe the behavior of the time series are not known and have to be established through a time-consuming procedure of trial and error and application of statistical tests. Furthermore, the linear structure of the model is not able to represent possible nonlinearities, which leads to inadequate forecasting performance. In order to 
cope with this issue, nonlinear methodologies, such as the nonlinear ARMA models were developed. However, the use of such models necessitates the determination of the form of nonlinearity through an additional trial and error procedure. A solution to this problem has been given by the utilization of Artificial Neural Networks (ANNs), which are generic modelling structures that can capture any nonlinear behavior.

A comparison between linear models and ANNs has been conducted by Agrawal \& Schorling (1996) who studied problems concerning sales of food products. They found that in many cases ANNs performed better than linear models. A comprehensive review of the literature on the utilization of ANNs in forecasting problems in various areas was later presented by Zhang et al. (1998), where in $30 \%$ of the cases studied, ANNs performed equally well with linear methods and better in $56 \%$. In another comparative study of the forecasting performance of linear and nonlinear methods, Stock and Watson (1999) found that combinations of nonlinear methods are better than combinations of linear methods and observed that feedforward neural networks, (FNNs) performed equally well or better than traditional methods in more than half of the cases. An important contribution to the comparison of the available forecasting methodologies is the series of M-Competitions. In the latest one, the M3-Competition (Makridakis and Hibon 2000), the most popular forecasting methodologies and commercial software were examined in several test cases. The neural network methods that were applied to these data did not perform well; that was attributed by Balkin (2001) to the characteristics of the time series studied: only $25 \%$ of the data sets exhibited strong nonlinear character, 
while in most cases the series contained an insufficient number of data points for model building.

As mentioned before, typical MPC configurations are based on the current estimation of the disturbance, which is projected without changes throughout the prediction horizon. In this paper we propose the incorporation of a Radial Basis Function (RBF) neural network time series model into the MPC framework, which serves as an advanced nonlinear forecasting mechanism concerning future demand. The proposed scheme can improve significantly the performance of the system, since more information is provided to the formulation of the MPC optimization problem that is solved in real time. We should note here that in most dynamical systems it is not possible to develop a time series model that can predict the future behavior of the disturbance, which typically follows a random trend. Thus, the particular MPC framework developed in this paper is devoted to production-inventory systems.

\section{Development of the Time Series Forecasting Model}

A general form of the $\operatorname{ARIMA}(p, d, q)$ model is the following:

$$
\left(1-\sum_{i=1}^{p} \varphi_{i} Z^{i}\right)(1-Z)^{d} X_{t}=\left(1+\sum_{i=1}^{q} \theta_{i} Z^{i}\right) \varepsilon_{t}
$$

where $Z$ is the lag operator, $\varphi_{\mathrm{i}}$ are the parameters of the autoregressive part of the model, $\theta_{\mathrm{i}}$ are the parameters of the moving average part, $p$ is the order of autoregression, $d$ is the 
order of differencing, $q$ is the order of the moving average process and $\varepsilon_{\mathrm{t}}$ are error terms. Depending on the values of the parameters in the general form depicted in Eq. (1), there are many types of ARIMA models, like the Autoregressive (AR) model, which is an $\operatorname{ARIMA}(p, 0,0)$ model where only past values of the function are used to produce a forecast.

ANNs are complex, generic nonlinear architectures, whose inherent sophisticated structure allows them to mimic nonlinear dynamic behaviors. The Radial Basis Function $(\mathrm{RBF})$ neural network is a special architecture that consists of three layers, as shown in Figure 1. The input layer is used to feed the input vector $\mathrm{x}$ into the model. The hidden layer contains a number of nodes. Each hidden node is associated with a vector (center) c whose dimension is equal to dimension of the input vector. The hidden nodes apply nonlinear transformation on the Euclidean distances between the input vector and the node centers, using radial basis functions. In the present work, the thin-plate-spline radial basis function is employed. The output layer serves as a linear summation unit:

$$
\hat{y}=\sum_{j=1}^{L} w_{j} f\left(\left\|\mathbf{x}-\mathbf{c}_{j}\right\|\right)
$$

In the above equation $\mathrm{c}_{j}$ is the center of the $j$ th hidden node, $w_{j}$ is the connection weight between the $j$ th hidden node and the output node, $f$ is the radial basis function, $L$ is the number of nodes in the hidden layer, $\|\square\|$ is the Euclidean norm and $\hat{y}$ is the estimated output value. In the case of sales forecasting, the input data vector $\mathbf{x}$ contains past sales 
figures and perhaps other variables that affect sales, while the output variable $\hat{y}$ is the estimated future demand.

Insert Figure 1 around here

A training algorithm for RBF networks is based on the use of a set of input-output data $\left(\mathrm{x}_{i}, y_{i}\right), \mathrm{i}=1,2, \ldots, K$ in order to determine the structure and the parameters of the network that lead to a minimum error between the predicted output and the actual values. This defines a Mixed Integer Nonlinear Programming (MINLP) optimization problem, where the following objective function is minimized:

$$
\begin{aligned}
& J\left(L, \mathbf{c}_{j}, w_{j}\right)=\sum_{i=1}^{K}\left(y_{i}-\hat{y}_{i}\right)^{2}(3), \\
& \text { where: } \quad \hat{y}_{i}=\sum_{j=1}^{L} w_{j} f\left(\left\|\mathbf{x}_{i}-\mathbf{c}_{j}\right\|\right), \quad i=1,2, \ldots, K
\end{aligned}
$$

\footnotetext{
Special training algorithms that take advantage of the RBF model structure are employed to solve this optimization problem. In our work we used the fuzzy means clustering technique (Sarimveis et al. 2002), which is able to determine the structure of the model without trial and error or user interaction. Furthermore, this specific technique has proven to be orders of magnitude faster compared to alternative RBF training algorithms.
} 
A central issue in the construction of a forecasting model is the selection of the dataset on which it will be based. There might be several variables available for the process under study. However, some of them may not affect the time series significantly. In this case, inclusion of all the available variables results to a model which is unnecessarily large in size and perhaps poor as far as accuracy is concerned. Furthermore, there might be variables that do affect the time series, but they are highly correlated with each other. Therefore, it is essential to employ some form of selection procedure in order to sort out the most important variables. One approach is to employ multivariate analysis tests, such as the partial least squares (PLS) (Wold et al. 2001) or the principle component analysis (PCA) (Hörnquist et al. 2003). However, both these methods produce latent (artificial) variables and, more importantly, there is no clear connection to the physical variables of the problem studied. Another alternative approach to this issue, often used in marketing research, is to utilize statistical tests for variable selection (i.e. Poh et al. 1998). Additionally, artificial intelligence technologies like fuzzy logic in combination with statistical methodologies have been employed to the variable selection problem (Mastorocostas et al. 2001). A variable selection method must therefore find the desired balance between two conflicting objectives: Minimizing modeling error while at the same time minimizing the number of selected variables, which is translated to balancing the model's accuracy and simplicity. There are standard selection criteria that have been employed for this purpose, such as the final prediction error (FPE) (Akaike 1970), Akaike's information criterion (AIC) (Akaike 1974), and the modified FPE (Leontaritis and Billings 1987). 


\section{The proposed integrated MPC - Time Series Forecasting method}

MPC is nowadays recognized as a standard methodology for the control of industrial and process systems (Morari and Lee 1999). This is because of its capability to incorporate constraints for the manipulated and/or the controlled variables, to handle the nonlinearities which are often present in dynamical systems and to overcome modelling mismatch. The idea of model predictive control is simple and is graphically presented in Figure 2. A process model is used to predict the effect of a finite number of future moves on the controlled variables. This model is incorporated in an on-line open loop optimization problem, which determines the optimal control sequence for a given performance criterion. The simplest MPC objective function is the weighted sum of the two basic targets, namely the sum of squared differences between the predicted outputs and their set points over the future prediction horizon $p h$ and the sum of squares of the control moves over the control horizon $c h$. After the solution of the minimization problem is found, only the first of the future control actions is implemented to the system. The same procedure is performed repetitively at each time step. In the rest of this section, the proposed control methodology will be described in detail. Figure 3 presents the block diagram of the control scheme, consisting of the process, the Model Predictive Controller and the demand forecasting mechanism.

Insert Figure 2 around here

Insert Figure 3 around here 


\subsection{Process Model and Material Balance}

In many production-inventory systems, the production process is modelled by a pure delay unit, with a discrete transfer function equal to $z^{-T}$, where $T$ is the lead time. However, such an assumption is not always realistic. In this work, we assume that the process dynamic behavior is described by a Finite Impulse Response (FIR) model. In this case, the system output (production rate $R(t)$ ) will be given by the following equation:

$R(t)=\sum_{i=1}^{n} g_{i} \cdot \operatorname{Order}(t-i)$

where $\operatorname{Order}(t-i), i=1, \ldots, n$ is the order rate at discrete time instance $t-i, n$ is the system order and $g_{i}, i=1, \ldots, n$ are the system parameters. Eq. (5) can easily lead to the transfer function between production rate and order rate $z$-transformed signals:

$\frac{R(z)}{\operatorname{Order}(z)}=g_{1} \cdot z^{-1}+\ldots+g_{n} \cdot z^{-n}$

which obviously is a generalization of pure delay.

From the block diagram of Figure 3, inventory $\operatorname{Inv}(z)$ is given by the following equation: 


$$
\operatorname{Inv}(z)=\frac{1}{1-z^{-1}}(R(z)-\operatorname{Sales}(z))
$$

where $\operatorname{Sales}(z)$ is the $z$-transform of customers demand time signal Sales $(t)$, while $\frac{1}{1-z^{-1}}$ represents the integration dynamics in the $z$-domain, assuming that the sampling time is equal to one time unit. Combining Eqs. (5)-(7) we arrive to the following result, which shows that the inventory level at time $t$ is related to the order signal with an autoregressive with exogenous input model (ARX) that also considers customer demand as an external measured disturbance:

$$
\operatorname{Inv}(t)=\operatorname{Inv}(t-1)+\sum_{i=1}^{n} g_{i} \cdot \operatorname{Order}(t-i)-\operatorname{Sales}(t)(8)
$$

\subsection{Robust Model Predictive Control scheme}

In the proposed inventory control scheme (Figure 3), the manipulated variables are the order rates $\operatorname{Order}(t+j \mid t), j=0, \ldots, c h$, while the predicted inventory level $\hat{\operatorname{Inv}}(t+j \mid t), j=1, \ldots, p h$ is considered as the controlled variable. A predictor for inventory is formulated based on the material balance represented by Eq. (8). In order to test the robustness of the proposed control scheme, we assume that the predictor is based on an approximation of the process parameters $\hat{g}_{i}, i=1, \ldots, n$ and not on their true values. 
The predictor incorporates the demand forecasting strategy which provides a prediction of the unknown future sales over the prediction horizon, ForSales $(t+j \mid t), j=1, \ldots, p h$. As mentioned in the previous section, this strategy can range from a simple projection of current sales to sophisticated time series forecasting models. The optimization problem solved on line is described by the following set of Eqs. (9)-(17).

$\min _{\operatorname{Order}(t+i), i=0, \ldots, c h} \sum_{j=1}^{p h}(w(\hat{\operatorname{In} v}(t+j \mid t)-\operatorname{TIn} v))^{2}+\sum_{j=0}^{c h-1}(r \cdot \delta \operatorname{Order}(t+j \mid t))^{2}$

subject to:

$$
\begin{aligned}
& \hat{\operatorname{Inv}}(t+j \mid t)=\hat{\operatorname{Inv}}(t+j-1 \mid t)+\sum_{i=1}^{n} \hat{g}_{i} \cdot \operatorname{Order}(t+j-i \mid t)-\text { ForSales }(t+j \mid t)+e(t+j \mid t), \\
& j=1, \ldots, p h(10) \\
& \hat{\operatorname{Inv}}(t \mid t)=\operatorname{Inv}(t) \\
& e(t+j \mid t)= \begin{cases}e(t \mid t), & \text { if } j=1 \\
0, & \text { else }\end{cases} \\
& e(t \mid t)=\operatorname{Inv}(t)-\operatorname{Inv}(t-1)-\sum_{i=1}^{n} \hat{g}_{i} \cdot \operatorname{Order}(t-i)+\operatorname{Sales}(t) \\
& \delta \operatorname{Order}(t+j \mid t)=\operatorname{Order}(t+j \mid t)-\operatorname{Order}(t+j-1 \mid t), \quad j=0, \ldots, c h \\
& u_{\min } \leq \operatorname{Order}(t+j \mid t) \leq u_{\max }, \quad j=0, \ldots, c h \\
& \delta u_{\min } \leq \delta \operatorname{Order}(t+j \mid t) \leq \delta u_{\max }, \quad j=0, \ldots, c h \\
& \delta \operatorname{Order}(t+j \mid t)=0, \quad j=c h+1, \ldots, p h
\end{aligned}
$$


where $\operatorname{Inv}(t+j \mid t), j=1, \ldots, p h$ is the $j$ - step ahead prediction of inventory level, TInv is the target inventory value, $\delta \operatorname{Order}(t+j \mid t), j=0, \ldots, c h$ are the future control moves, $w, r$ are weight factors balancing the two conflicting objectives in Eq. (9) and $e(t+j \mid t), j=1, \ldots, p h$ is the estimated error. Eq. (11) shows that the current predicted inventory value is set equal to the actual inventory level. Eq. (12) denotes that the current error as it is estimated from Eq. (13) should be added only on the first future inventory level prediction. Eqs. (15)-(16) are hard constraints that limit the manipulated variables and the control moves between upper and lower bounds. $u_{\min }, u_{\max }$, are the lower and upper bounds for order rates and $\delta u_{\min }, \delta u_{\max }$, are the lower and upper bounds for control moves. Finally Eq. (17) denoted that no control moves are allowed after the end of the control horizon.

\section{Results}

The proposed MPC-forecasting framework was applied to industrial data, in order to assess its effectiveness on real-life cases. For the purpose of evaluating the efficiency of the combined MPC-forecasting systems with respect to the forecasting strategy that is adopted, three additional forecasting methods were applied to the system alongside with the proposed neural network methodology. The forecasting methodologies that were tested are the following: typical MPC forecasting (projection of current sales throughout the prediction horizon), a Linear Autoregression (Linear AR) time series forecasting 
model, a Holt-Winters forecasting model and the proposed RBF neural network (RBF ANN) forecasting model.

Sales data were supplied by a leading Greek dairy products manufacturer and concern the daily sales of a fast moving product. A specially designed genetic algorithm (GA) was used for the selection of the most appropriate input variables to the model, based on the FPE criterion. The algorithm employs a hybrid coding of genes. Each potential input variable is coded by a binary gene denoting whether the variable is present in the model (the gene has the value 1) or not (the gene has the value 0). An additional integer gene in each chromosome corresponds to the number of fuzzy sets that are defined in the domain of each variable, which is a parameter used by the fuzzy means algorithm. The standard crossover operator and a specially designed mutation operator are used to produce new generations of solutions. The candidate variables were sales on the 6 previous days of the current year, sales on the corresponding day and the 6 previous days of last year and the percentile change in sales between the current year and the previous year. The input variables chosen by the GA were five: the sales of the current year with lags -1 and -6 and the sales of the previous year with lags $-3,-5$ and -6 . In Doganis et al. (2006), the development of the time series forecasting models is presented in details. Figure 4 shows the discrepancies between the true sales trend and the forecasts produced by the linear $\mathrm{AR}$ and the nonlinear RBF time series forecasting models. The average forecasting errors for all four strategies are summarized in the first column of Table 1, where it is obvious that the nonlinear RBF method provides better forecasts than the rest of the forecasting strategies. 


\section{Insert Figure 4 around here \\ Insert Table 1 around here}

The effect of the accuracy of the demand forecasting strategy on the performance of the proposed MPC production-inventory control scheme will be examined next.

The production dynamics are described by the following FIR model:

$$
\begin{aligned}
R(t)=0.05 \cdot \operatorname{Order}(t-1)+0.25 \cdot \operatorname{Order}(t-2) & +0.25 \cdot \operatorname{Order}(t-3)+0.20 \cdot \operatorname{Order}(t-4) \\
& +0.15 \cdot \operatorname{Order}(t-5)+0.10 \cdot \operatorname{Order}(t-6)
\end{aligned}
$$

In order to examine the robustness of the method, we assume that there is a mismatch between the true dynamics and the available model:

$$
\begin{aligned}
\hat{R}(t)=0.10 \cdot \operatorname{Order}(t-1)+0.20 \cdot \operatorname{Order}(t-2) & +0.30 \cdot \operatorname{Order}(t-3)+0.20 \cdot \operatorname{Order}(t-4) \\
& +0.10 \cdot \operatorname{Order}(t-5)+0.10 \cdot \operatorname{Order}(t-6)
\end{aligned}
$$

The rest of the MPC parameters are provided in Table 2. The results are summarized in the second column of Table 1, where the sums of the squared deviations from the desired inventory set point are presented for all forecasting strategies. It is apparent that as the accuracy of the forecasting model increases, the performance of the MPC module is improved. In particular, the forecasting method that produced the best results, namely the RBF time series model led to a drastic reduction of the deviation from the inventory set point.

Insert Table 2 around here 
Figs. 5-6 compare the inventory trajectory and the corresponding control actions (order levels) using the typical MPC forecasting strategy and the linear AR time forecasting model. The importance of the adopted forecasting strategy is obvious. The Linear AR time series forecasting model produces a considerably less oscillatory inventory trajectory compared to the typical MPC forecasting strategy. At the same time the control moves are less aggressive. However, in both forecasting schemes, the average inventory level is clearly higher compared to the desired set point. This is corrected by using the more sophisticated and accurate RBF forecasting strategy as shown in Figs 7-8 which now compare the performances of the linear AR and the ANN time forecasting models. With small control action modifications (Figure 7), the MPC configuration employing the RBF time series forecasting model is able to produce an inventory trajectory which is similar to the one produced by the linear AR model as far as variation is concerned, but with a much closer average to the desired set point.

Insert Figure 5 around here

Insert Figure 6 around here

Insert Figure 7 around here

Insert Figure 8 around here

\section{Conclusion}

A framework for supply chain management based on Model Predictive Control combined with a forecasting module was presented. Various linear and nonlinear forecasting methodologies were evaluated in order to investigate the existence of possible nonlinearity in the sales time series. The nonlinear method used, namely the RBF neural 
network model, exhibited superior forecasting performance on the time series examined and the corresponding MPC scheme was the most efficient as far as the inventory control problem is concerned. The results demonstrate that a higher forecasting accuracy visibly leads to improved control performance, thus contributing to more efficient management of the supply chain.

\section{Acknowledgements}

This work was performed under the measure 8.3 of the operational program "Competitiveness" of the $3^{\text {rd }}$ Community Support Program and was co-funded by $75 \%$ from the European Union- European Social Fund and by $25 \%$ from the General Secretariat of Research and Technology in Greece. 


\section{References}

Agrawal, D., Schorling, C., Market share forecasting: An empirical comparison of artificial neural networks and multinomial logit model. Journal of Retailing, 1996, 72(4), 383-407.

Akaike, H., Statistical predictor identification. Annals of the Institute of Statistical Mathematics, 1970, 22, 203-217.

Akaike, H., A new look at a statistical model identification. IEEE Transactions on Automatic Control, 1974, 19, 716-722.

Balkin, S., The value of nonlinear models in the M3-Competition (Commentaries on the M3-Competition). International Journal of Forecasting, 2001, 17, 545-546.

Box, G. E. P., Jenkins, G. M., Reinsel, G. C., Time series analysis: forecasting and control, 1994, (Prentice Hall: Englewood Cliffs, New Jersey).

Braun, M. W., Rivera, D. E., Flores, M. E., Carlyle, W. M., Kempf, K. G., A model predictive control framework for robust management of multi-product, multiechelon demand networks. Annual Reviews in Control, 2003, 27, 229-245.

Doganis, Ph., Alexandridis A., Patrinos P., Sarimveis, H., Time series sales forecasting for short shelf-life products based on artificial neural networks and evolutionary computing. Journal of Food Engineering, 2006, 75, 196-204.

Hörnquist, M., Hertz, J., \& Wahde, M., Effective dimensionality for principal component analysis of time series expression data. Biosystems, 2003, 71(3), 311-317.

Kapsiotis, G., Tzafestas, S. , Decision making for inventory/production planning using model-based predictive control. In Parallel and distributed computing in 
engineering systems, edited by Tzafestas, S., Borne, P., Grandinetti, L., pp. 551556, 1992 (Elsevier: Amsterdam).

Leontaritis, I. J., Billings, S. A., Model selection and validation methods for non-linear systems. International Journal of Control, 1987, 45, 311-341.

Lin, P. H., Jang, S. S., Wong, D. S. H., Predictive control of a decentralized supply chain unit. Industrial Engineering \& Chemistry Research, 2005, 44, 9120-9128.

Makridakis, S., Hibon, M., The M3-Competition: Results, conclusions and implications. International Journal of Forecasting, 2000, 16 (4), 451-476.

Mastorocostas, P. A., Theocharis, J. B., Petridis, V. S., A constrained orthogonal leastsquares method for generating TSK fuzzy models: Application to short-term load forecasting. Fuzzy Sets and Systems, 2001, 118, 215-233.

Mestan, E., Türkay, M., \& and Arkun, Y, Optimization of Operations in Supply Chain Systems Using Hybrid Systems Approach and Model Predictive Control. Ind. Eng. Chem. Res. 2006, 45, 6493-6503

Morari, M., Lee, J. H., Model predictive control: Past, present, and future. Computers \& Chemical Engineering, 1999, 23, 667-682.

Perea Lopez, E., Ydstie, B. E., Grossmann, I., A model predictive control strategy for supply chain management. Computers \& Chemical Engineering, 2003, 27, 12011218.

Poh, H. L., Yao, J., Jašic, T., Neural networks for the analysis and forecasting of advertising and promotion impact. International Journal of Intelligent Systems in Accounting, Finance \& Management, 1998, 7, 253-258. 
Sarimveis, H., Alexandridis, A., Tsekouras, G., Bafas, G., A fast and efficient algorithm for training radial basis function neural networks based on a fuzzy partition of the input space. Industrial and Engineering Chemistry Research, 2002, 41, 751-759.

Stock, J. H., Watson, M. W., A comparison of linear and non-linear university models for forecasting economic time series. In Cointegration, causality, and forecasting: A Festschrift in honour of Clive W.J. Granger., edited by Engle R. F. \& White H., Wold, S., Sjöström, M., \& Eriksson, L., PLS-regression: A basic tool of chemometrics. Chemometrics and Intelligent Laboratory Systems, 2001, 58(2), 109-130.

Zhang, G. P., Time series forecasting using a hybrid ARIMA and neural network model. Neurocomputing, 2003, 50, 159-175. 


\section{FIGURES}

Figure 1. The RBF neural network architecture.

Figure 2. MPC basic concept

Figure 3. Block diagram of the proposed MPC configuration for inventory control

Figure 4. Actual sales and the forecasts produced by the Linear AR and RBF time series forecasting models.

Figure 5. MPC performance: Production orders using the typical MPC and the linear AR forecasting methods

Figure 6. MPC performance: Inventory trajectories using the typical MPC and the linear AR forecasting methods

Figure 7. MPC performance: Production orders using the linear AR and the proposed RBF forecasting methods

Figure 8. MPC performance: Inventory trajectories using the linear AR and the proposed RBF forecasting methods 


1
2
3
4
5
6
7
8
9
10
11
12
13
14
15
16
17
18
19
20
21
22
23
24
25
26
27
28
29
30
31
32
33
34
35
36
37
38
39
40
41
42
43
44
45
46
47
48
49
50
51
52
53
54
55
56
57
58
60

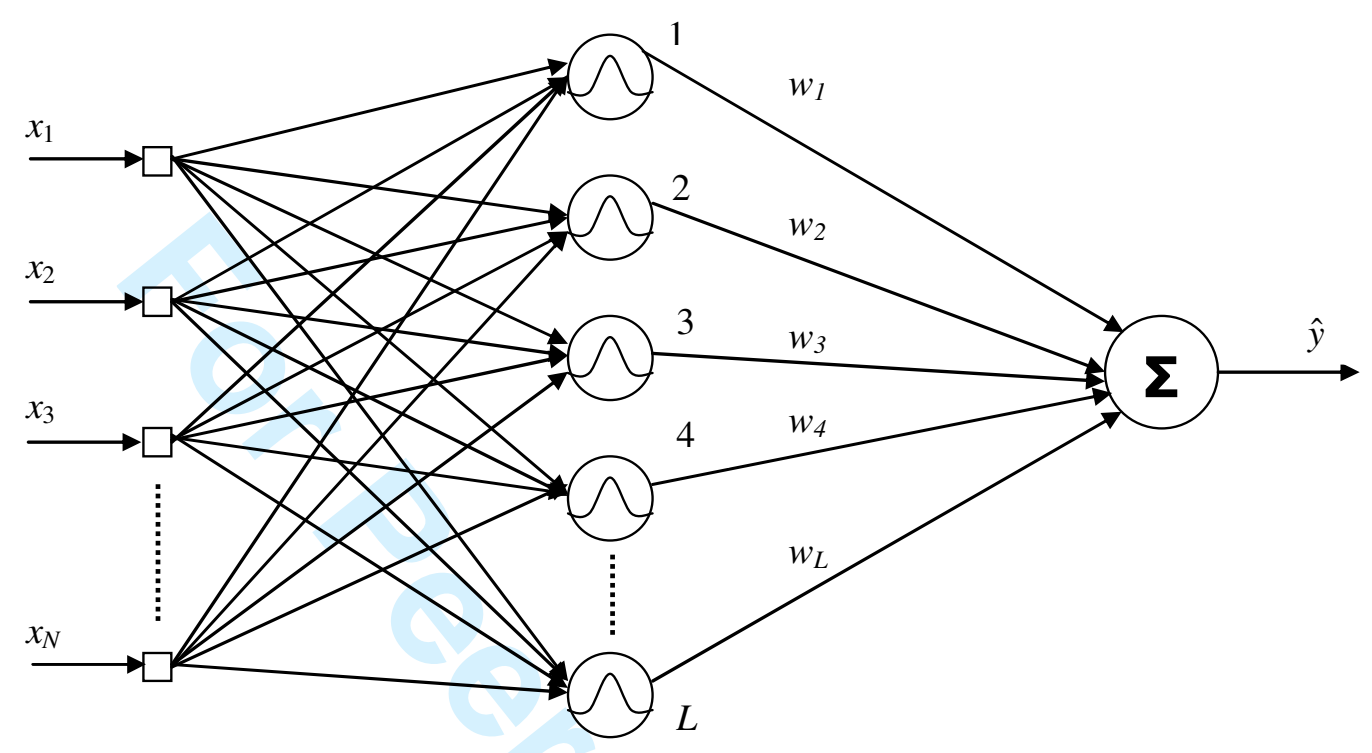

Figure 1 


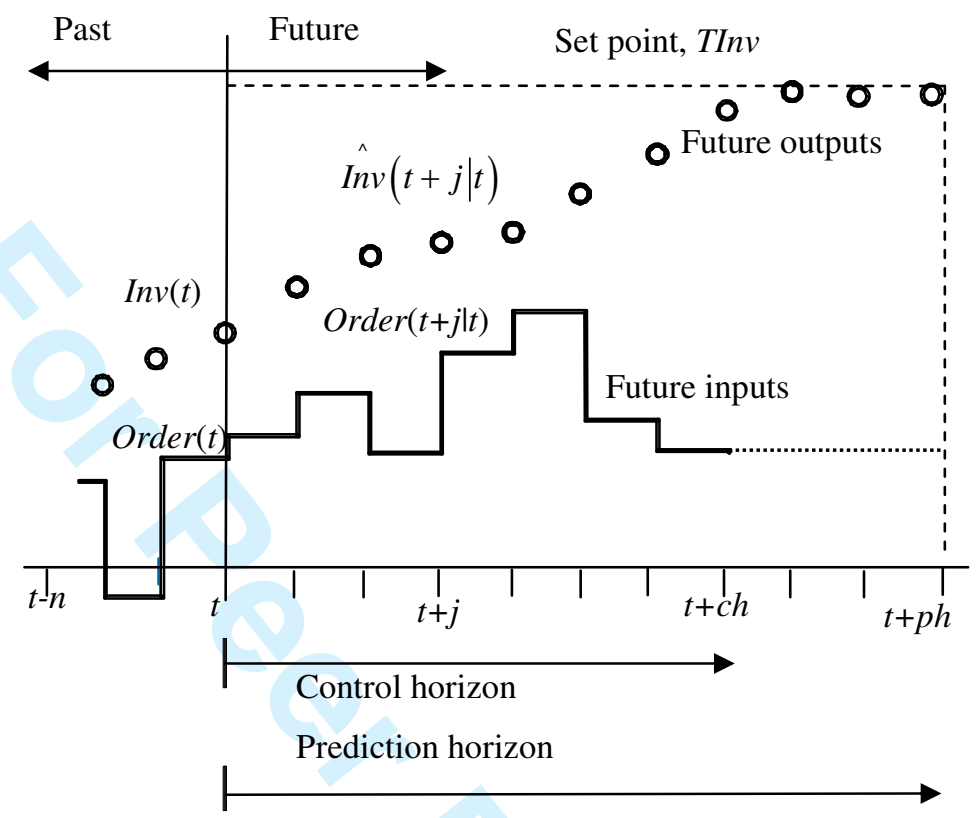

Figure 2 
1

2

3

4

5

6

7

8

9

10

11

12

13

14

15

16

17

18

19

20

21

22

23

24

25

26

27

28

29

30

31

32

33

34

35

36

37

38

39

40

41

42

43

44

45

46

47

48

49

50

51

52

53

54

55

56

57

58

59

60

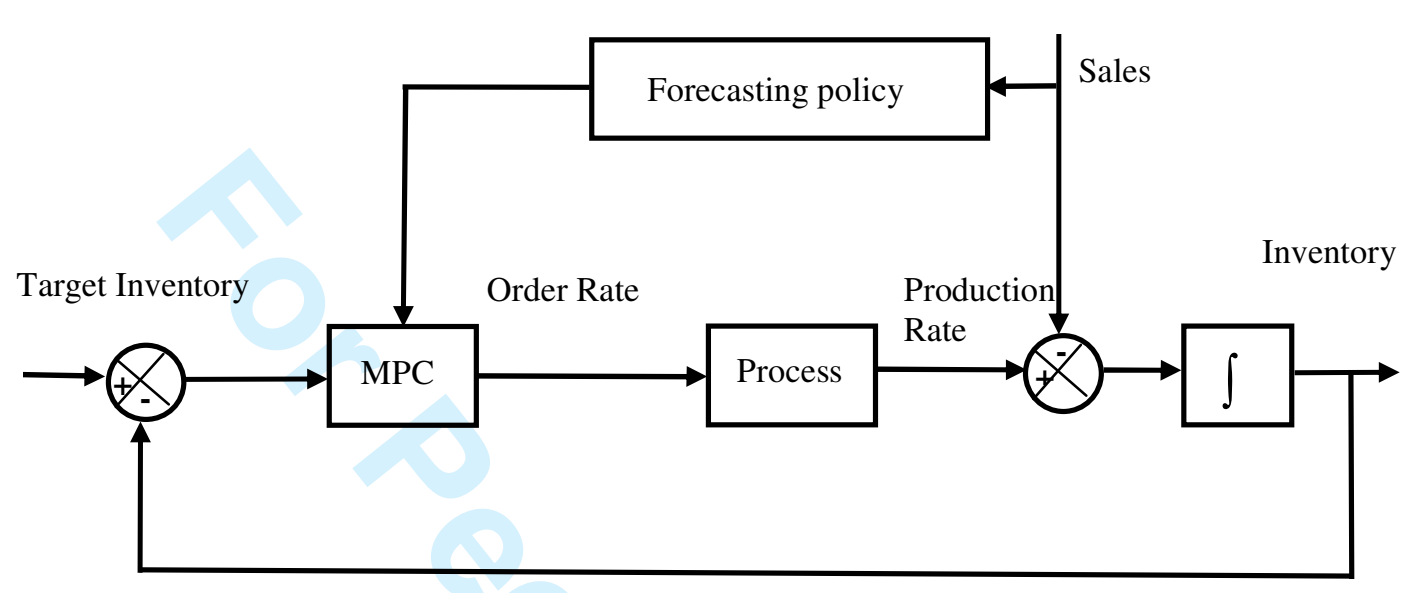

Figure 3 


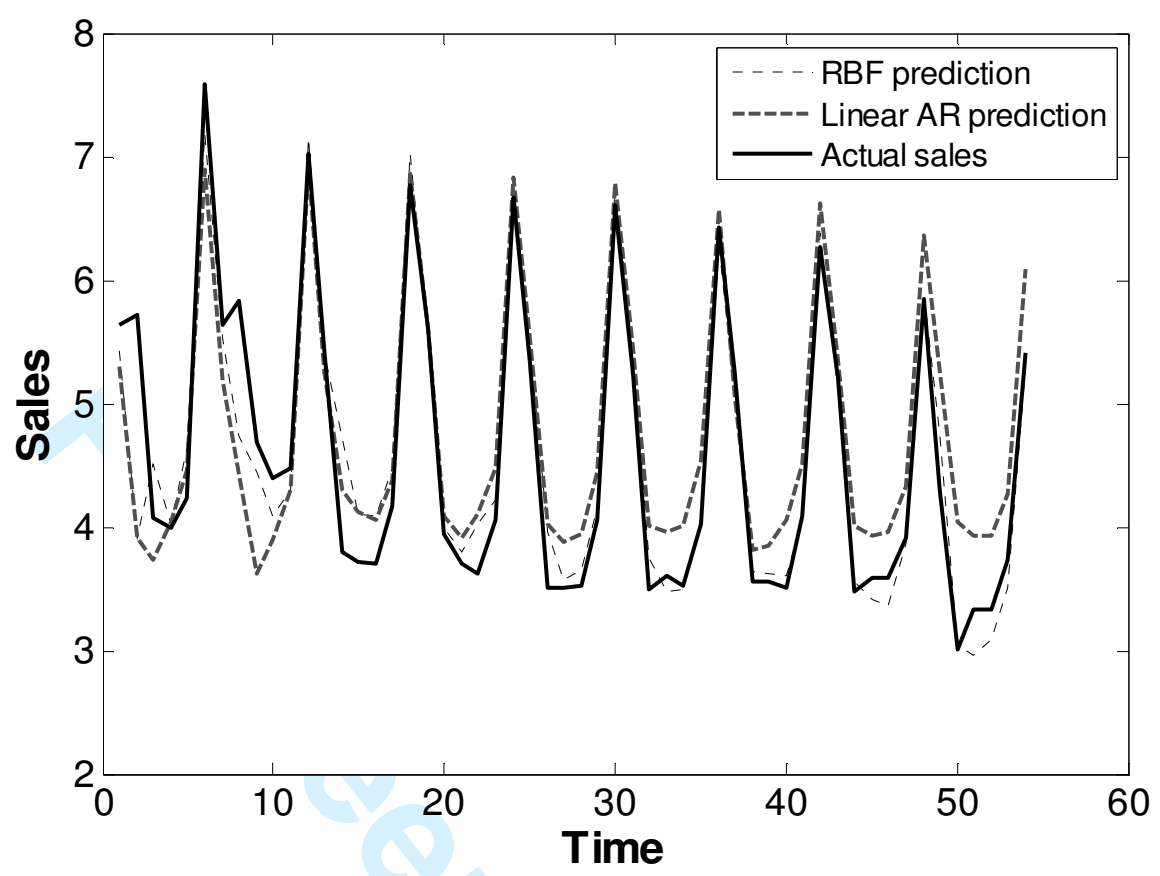

Figure 4 


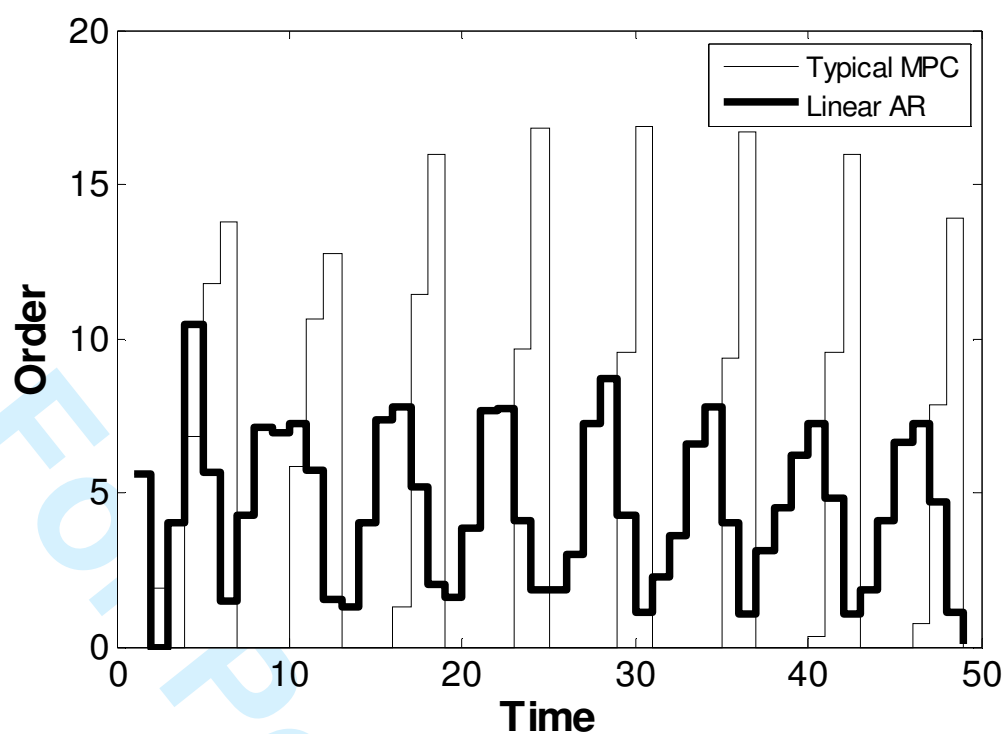

Figure 5 


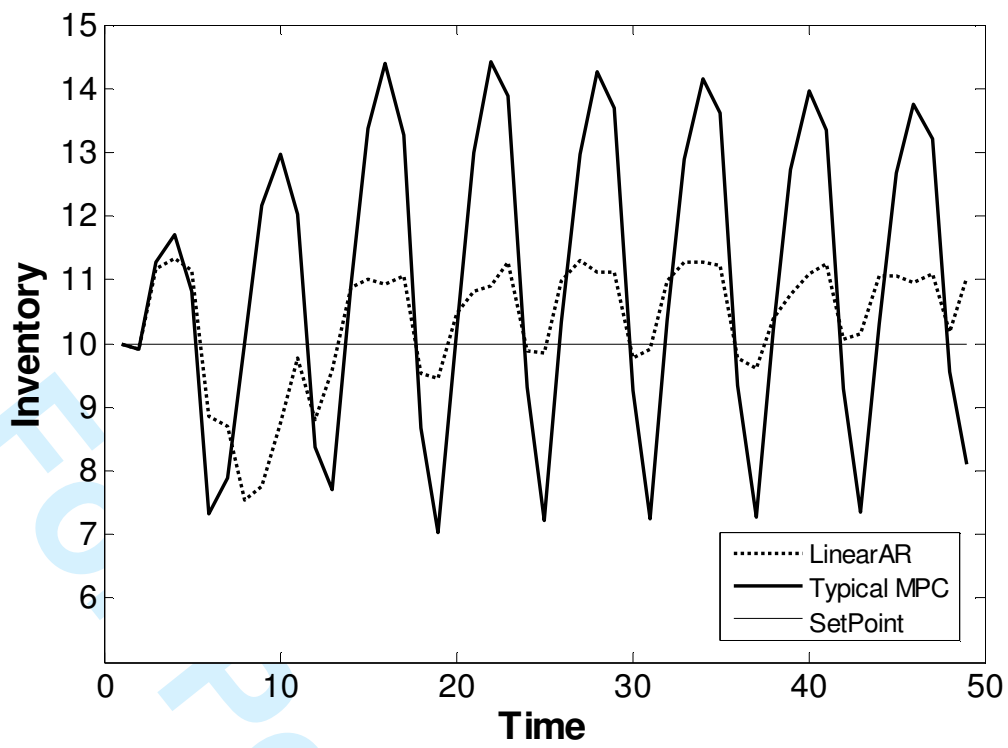

Figure 6

http://mc.manuscriptcentral.com/tprs Email: ijpr@lboro.ac.uk 


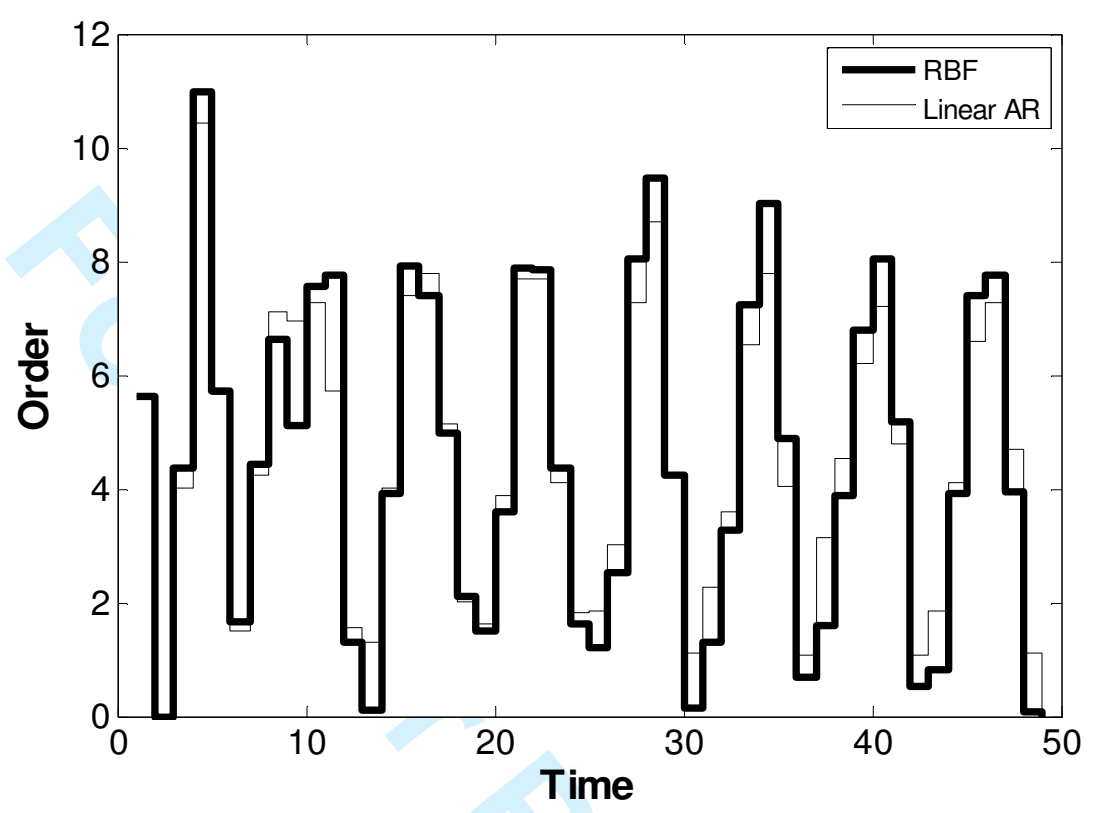

Figure 7

http://mc.manuscriptcentral.com/tprs Email: ijpr@lboro.ac.uk 


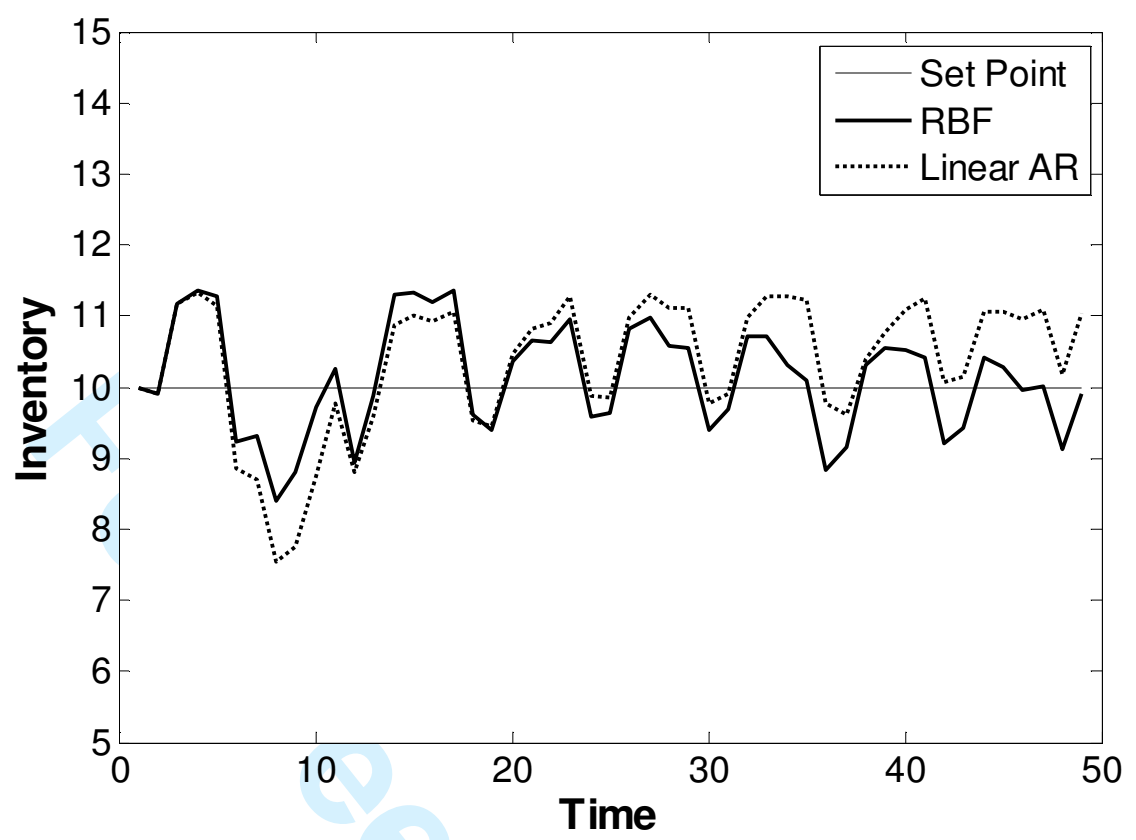

Figure 8 
1

2

3

4

5

6

7

8

10

11

12

13

14

15

16

17

18

19

20

21

22

23

24

25

26

27

28

29

30

31

32

33

34

35

36

37

38

39

40

41

42

43

44

45

46

47

48

49

50

51

52

53

54

55

56

57

58

59

60

\section{TABLES}

Table 1. Implementation results

Table 2. MPC parameters 


\begin{tabular}{ccc}
\hline \multicolumn{2}{c}{ Table 1. Implementation results } \\
\hline Method & Average Forecasting Error (\%) & $\begin{array}{c}\text { Deviation from inventory set point } \\
\text { (Sum of Squared Errors, SSE) }\end{array}$ \\
\hline RBF & 5.34 & 29.44 \\
Holt- Winters & 9.58 & 47.43 \\
Linear AR & 10.20 & 49.62 \\
Typical MPC & 19.88 & 319.77 \\
\hline
\end{tabular}


Table 2. MPC parameters

\begin{tabular}{cc}
\hline Parameters & Values \\
\hline$n$ & 6 \\
$c h$ & 4 \\
$p h$ & 5 \\
$w$ & 60 \\
$r$ & 1 \\
$u_{\min }$ & 0 \\
$u_{\max }$ & 100 \\
$\delta u_{\min }$ & -100 \\
$\delta u_{\max }$ & 100 \\
$T I n v$ & 10 \\
\hline
\end{tabular}

32

33

34

35

37

38

39

40

41

42

44

45

46

47

48

50

51

52

53

54

55

56

57

59

60 